Revisión

\title{
Logros, retos y perspectivas de la investigación en mejoramiento genético de bovinos productores de carne en el INIFAP
}

Ángel Ríos Utrera ${ }^{a}$

Guillermo Martínez Velázquez ${ }^{\text {b }}$

René Calderón Chagoya ${ }^{c}$

Moisés Montaño Bermúdez ${ }^{\mathrm{c}}$

Vicente Eliezer Vega Murillo ${ }^{d *}$

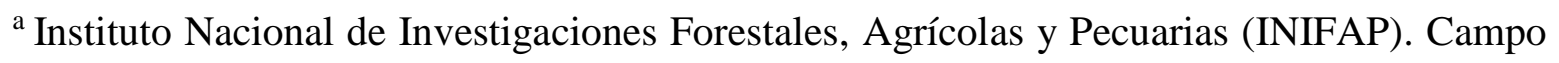
Experimental La Posta, km 22.5 carretera federal Veracruz-Córdoba, Paso del Toro, Municipio de Medellín, 94277. Veracruz, México.

${ }^{\mathrm{b}}$ INIFAP. Sitio Experimental El Verdineño. Nayarit, México.

${ }^{c}$ INIFAP. Centro Nacional de Investigación Disciplinaria en Fisiología y Mejoramiento Animal. Querétaro, México.

${ }^{\mathrm{d}}$ Universidad Veracruzana. Facultad de Medicina Veterinaria y Zootecnia. Veracruz, México.

*Autor de correspondencia: vvega@uv.mx

\section{Resumen:}

El objetivo fue presentar resultados de investigación sobre mejoramiento genético de bovinos productores de carne realizados por el Instituto Nacional de Investigaciones Forestales, Agrícolas y Pecuarias, así como los retos y perspectivas de investigación a corto, mediano y largo plazo en dicha área del conocimiento. Se utilizaron tesis de maestría, trabajos de congresos y artículos científicos publicados de 1987 a 2020. En los últimos 34 años, se logró caracterizar productiva y reproductivamente animales Bos indicus y Bos taurus x Bos indicus 
en condiciones tropicales, implementar evaluaciones genéticas multirraciales para las poblaciones Simmental-Simbrah y Charolais-Charbray, determinar la importancia de los efectos maternos en características de crecimiento y estimar la heredabilidad y correlaciones genéticas para características de crecimiento y reproductivas de machos y hembras en animales Bos taurus y Bos indicus, comprobar que la interacción genotipo-ambiente es un factor importante en la expresión de peso al destete en Simmental, desarrollar factores de ajuste de edad de la madre para pesos al nacimiento y destete, desarrollar un prototipo de evaluación genética nacional para permanencia productiva y otro para fertilidad de vaquillas, estimar la diversidad genética de las poblaciones Simmental, Charolais y Simbrah, e identificar SNP asociados a características de crecimiento en Simmental y Simbrah. En el corto plazo, se espera desarrollar índices de selección y predecir el mérito genético de características de canal; en el mediano, realizar evaluaciones genómicas para tolerancia al estrés calórico, consumo de alimento residual y características de salud; y a largo plazo, realizar predicciones genómicas a través de razas.

Palabras clave: Asociación genómica, Bovinos de carne, Correlaciones genéticas, Cruzamientos, Diversidad genética, Heredabilidad, Mejoramiento genético.

Recibido: 27/11/2020

Aceptado: 29/03/2021

\section{Introducción}

El propósito del cruzamiento entre razas en bovinos productores de carne es utilizar las diferencias entre razas y los efectos de heterosis para mejorar la producción. Se estima que la heterosis en un programa de cruzamiento puede incrementar la productividad del hato de vacas en $26 \%$, en promedio, en comparación con un programa similar usando animales puros. El beneficio mayor se tiene por el uso de vacas $\operatorname{cruzadas}^{(1,2,3)}$. El incremento en la productividad por cruzamientos depende de las razas utilizadas. A principios de los ochenta, los ganaderos mexicanos no utilizaban vacas cruzadas para producir becerros para engorda y la disponibilidad de toros de razas especializadas era limitada, sobre todo en las regiones tropicales. En 1978 el Gobierno Federal a través del Instituto Nacional de Inseminación Artificial y Reproducción Animal de la Secretaría de Agricultura y Recursos Hidráulicos, puso a disposición de los ganaderos semen de razas Bos taurus especializadas en la producción de carne (Angus, Chianina, Charolais, Hereford, Limousin, Simmental y Suizo Pardo Europeo) ${ }^{(4)}$.

Por otro lado, el mejoramiento genético resulta de la selección de animales superiores al promedio como padres de la siguiente generación. Para identificar los animales 
genéticamente superiores, es necesario que las asociaciones de criadores de bovinos de registro integren bases de datos que permitan la realización de evaluaciones genéticas nacionales. La primera evaluación genética nacional en bovinos productores de carne fue realizada en el 2001, por un grupo de investigadores del Instituto Nacional de Investigaciones Forestales, Agrícolas y Pecuarias (INIFAP), usando la base de datos de la Asociación Mexicana Simmental Simbrah (AMSS). Desde entonces, el INIFAP ha celebrado convenios con algunas asociaciones que le permiten el uso de sus bases de datos con fines de investigación.

Algunas características de importancia económica son complejas, o difíciles o caras de medir, como comportamiento reproductivo, eficiencia alimenticia y características de canal. Para estas características puede resultar muy difícil realizar evaluaciones genéticas convencionales, como las que se realizan para características de crecimiento. Sin embargo, como resultado de la secuenciación del genoma bovino se desarrollaron arreglos con miles de marcadores conocidos como SNP (polimorfismos de un solo nucleótido) que permiten desarrollar estudios para predecir el mérito genético ${ }^{(5)}$.

El objetivo es presentar resultados de estudios realizados en el INIFAP sobre mejoramiento genético de bovinos productores de carne, así como los retos y perspectivas de investigación a corto, mediano y largo plazo en dicha área de conocimiento.

\section{Resultados de cruzamientos entre razas Bos taurus y Bos indicus en el campo experimental Las Margaritas en clima subtropical cálido húmedo $\operatorname{Af}(\mathbf{c})$}

En un estudio que comparó el comportamiento reproductivo hasta el destete de vacas Cebú (C) apareadas con sementales Bos taurus (Bt), Angus (A), Charolais (Ch), Hereford (H) y Suizo Pardo (S), y Bos indicus (Bi), Brahman (B) e Indubrasil (I), se encontró que cuando los empadres fueron en primavera la tasa de destete de vacas apareadas con toros $\mathrm{H}$, B y S fue mayor, la de las apareadas con toros A y Ch intermedia y la de las apareadas con toros I menor $(64.3,60.7,59.2,56.1,52.7$ y $39.5 \%$, respectivamente), pero cuando fueron en otoño, la tasa de destete de las vacas apareadas con toros $\mathrm{H}$ fue mayor, la de las apareadas con toros $\mathrm{S}$ intermedia y la de las apareadas con toros $\mathrm{Ch}$, I, A y B menor (72.4, 56.0, 55.9, 49.8, 44.9 y $43.0 \%$, respectivamente). En general, la tasa de destete de las vacas apareadas con toros B e I fue 16.5 y 23.7 puntos porcentuales menor que la de las apareadas con toros H. Estos resultados indican que la selección de la raza de toro y la época del año en que se realizarán los empadres son consideraciones importantes cuando se plantean esquemas de cruzamiento para la producción comercial de becerros al destete ${ }^{(6)}$. 
Por otro lado, el análisis de información de apareamientos que incluyó hembras I y B, y cruzadas F1 A x C, H x C, Ch x C y S x C mostró que las F1, con excepción de la Ch x C, tuvieron un comportamiento reproductivo superior desde la gestación hasta el destete, comparado con el comportamiento reproductivo de las vacas I y B (Cuadro 1).

Cuadro 1: Medias de cuadrados mínimos de productividad de vacas F1 Bos taurus x Bos indicus apareadas con toros Bos taurus y de vacas Cebú apareadas con toros Bos indicus

\begin{tabular}{|c|c|c|c|c|c|c|c|c|}
\hline $\begin{array}{l}\text { Grupo genético } \\
\text { de la vaca }\end{array}$ & $\begin{array}{l}\text { TG } \\
(\%)\end{array}$ & $\begin{array}{l}\mathbf{T P}^{¥} \\
(\%)\end{array}$ & $\begin{array}{l}T^{¥} \\
(\%)\end{array}$ & $\begin{array}{l}\text { PT* } \\
\text { (kg) }\end{array}$ & $\begin{array}{l}\text { PD210' } \\
\text { (kg) }\end{array}$ & $\begin{array}{l}\text { PTL }{ }^{\wedge} \\
(\mathbf{k g})\end{array}$ & $\begin{array}{l}\text { PP2 }^{\mathbb{N}} \\
(\%)\end{array}$ & $\begin{array}{l}\text { PP5 }^{\circledR} \\
(\%)\end{array}$ \\
\hline Hereford x Cebú & $82.0^{\mathrm{a}}$ & $81.0^{\mathrm{a}}$ & $73.0^{\mathrm{a}}$ & $738.8^{\mathrm{ab}}$ & $164.0^{\mathrm{bc}}$ & $1008^{b}$ & $92.0^{\mathrm{b}}$ & $47.0^{\mathrm{b}}$ \\
\hline Suizo Pardo x Cebú & $79.0^{\mathrm{a}}$ & $76.0^{\mathrm{ab}}$ & $73.0^{\mathrm{a}}$ & $667.9^{\mathrm{bc}}$ & $185.0^{\mathrm{ab}}$ & $1256^{\mathrm{a}}$ & $75.0^{\mathrm{ac}}$ & $42.0^{\mathrm{b}}$ \\
\hline Charolais x Cebú & $65.0^{\mathrm{b}}$ & $67.0^{\mathrm{abc}}$ & $61.0^{\mathrm{ab}}$ & $777.3^{\mathrm{a}}$ & $178.0^{c}$ & $1012^{b}$ & $92.0^{\mathrm{b}}$ & $55.0^{\mathrm{bc}}$ \\
\hline Angus x Cebú & $85.0^{\mathrm{a}}$ & $80.0^{\mathrm{a}}$ & $74.0^{\mathrm{a}}$ & $871.8^{\mathrm{a}}$ & $191.0^{\mathrm{a}}$ & $1454^{\mathrm{a}}$ & $84.0^{\mathrm{ab}}$ & $62.0^{c}$ \\
\hline Brahman & $67.0^{\mathrm{b}}$ & $65.0^{\mathrm{bc}}$ & $56.0^{\mathrm{b}}$ & $521.1^{\mathrm{c}}$ & $113.0^{\mathrm{d}}$ & $902^{\mathrm{b}}$ & $66.0^{\mathrm{a}}$ & $32.0^{\mathrm{a}}$ \\
\hline Indubrasil & $66.0^{\mathrm{b}}$ & $56.0^{\mathrm{c}}$ & $48.0^{\mathrm{b}}$ & --- & --- & --- & --- & --- \\
\hline
\end{tabular}

Tasas de gestación (TG), parto (TP) y destete (TD), producción total de por vida (PT), peso al destete ajustado a 210 días de edad de las crías (PD210), producción total de leche (PTL) y permanencia productiva al segundo (PP2) y quinto parto (PP5).

${ }^{{ }}$Las vacas fueron apareadas con toros de la misma raza que el padre (Ríos et al ${ }^{(7)}$ ).

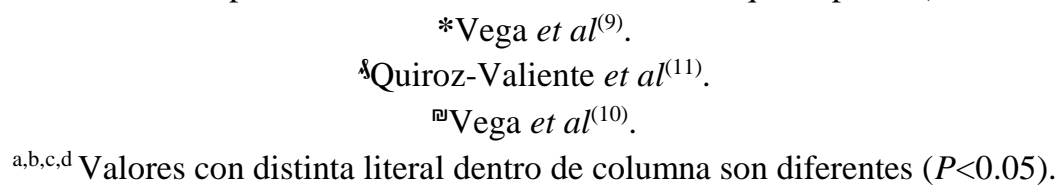

La superioridad de las vacas F1 en tasa de destete y peso al destete (PD) de sus crías sobre las vacas C, fue de 26 y 5.9 unidades porcentuales, respectivamente, lo que en términos de kilogramos de becerro destetado por vaca en empadre resultó en una ventaja total de $30.6 \%$. Similarmente, las vaquillas F1 tuvieron su primer parto a menor edad (17 meses en promedio) que las $\mathrm{Bi}^{(7)}$. Además, al estudiar las causas de desecho y la vida productiva, se encontró que el porcentaje de vacas desechadas por infertilidad fue mayor en las vacas Bi comparado con el de las F1, con promedios de 66.20 y $17.39 \%$, respectivamente. Los resultados indicaron que las vacas F1 tuvieron una vida productiva más larga que las Bi, debido a que la habilidad de las hembras cruzadas para permanecer más tiempo en el hato se debió a su mayor capacidad reproductiva, puesto que las diferencias en mortalidad y habilidad materna no fueron importantes. En conclusión, las vacas cruzadas F1 tuvieron una mayor vida productiva que las vacas puras $\mathrm{Bi}$; la diferencia promedio entre los dos grupos fue 2.8 años ${ }^{(8)}$.

Por otro lado, estudios adicionales permitieron evaluar la productividad total de por vida (PT) y la permanencia productiva (PP) a diferentes edades de vacas B y F1 A x C, S x C, Ch x C y $\mathrm{H}$ x $\mathrm{C}^{(9,10)}$. La PT se definió como el peso al destete acumulado de los becerros destetados hasta los 9 años de edad por vaca en empadre, mientras que PP se definió como la probabilidad de que una vaca tuviera 2 (PP2), 3 (PP3), 4 (PP4) o 5 (PP5) crías dado que había 
parido su primera cría antes de los 3 años de edad. Durante el desarrollo de los estudios, las vacas B se aparearon con toros B, mientras que las F1 se aparearon con toros Bi de 1986 a 1989 y con toros Bt de 1990 a 1994. En relación a la PT, las vacas F1 fueron superiores a las B en $101.2 \mathrm{~kg}$ a los 3 años de edad y en $242.9 \mathrm{~kg}$ a los 9 años de edad, observándose que a los 9 años de edad las vacas $\mathrm{A}$ x $\mathrm{C}$ y $\mathrm{Ch} \times \mathrm{C}$ acumularon significativamente más kilogramos de becerro destetado que las S x C y B (Cuadro 1) ${ }^{(9)}$. En cuanto a la PP, se encontraron diferencias importantes para PP2, con promedio de $92 \%$ para vacas Ch x C y H x C, y de 66 y $65 \%$ para vacas B y S x C, respectivamente; similarmente, se detectaron diferencias significativas para PP5, con promedios extremos de 62 y $32 \%$ para vacas A x C y B, respectivamente (Cuadro 1). Las vacas $\mathrm{Ch} \times \mathrm{C}, \mathrm{H}$ x C y $\mathrm{S} \times \mathrm{C}$ tuvieron un comportamiento intermedio. El estudio mostró que las vacas $\mathrm{F} 1 \mathrm{Bt} \times \mathrm{Bi}$ tuvieron mayor probabilidad de producir más crías que las vacas $\mathrm{B}^{(10)}$. Así, los dos estudios mencionados previamente reafirman la recomendación de utilizar vacas F1 como opción para incrementar la productividad hasta el destete en los sistemas vaca-cría de las regiones tropicales de México. En otra investigación se estimó la producción de leche de vacas B y de los cuatro grupos raciales F1 Bt x Bi y su correlación con el PD de las crías. Para medir la producción de leche, se utilizó la técnica de pesaje del becerro antes y después del amamantamiento. En el estudio se determinó un coeficiente de correlación entre producción total de leche y PD ajustado a 210 días de edad de 0.61. Además, se encontró que las vacas A x C y S x C tuvieron mayor producción total de leche y crías con mayor $\mathrm{PD}$ ajustado a 210 días de edad que las vacas $\mathrm{Ch}$ x C, H x C y B (Cuadro 1) ${ }^{(11)}$.

\section{Resultados de cruzamientos entre razas Bos taurus y Bos indicus en el campo experimental EI Macho en clima tropical cálido subhúmedo Aw}

Un estudio permitió evaluar las diferencias en el comportamiento productivo de crías $\mathrm{F} 1 \mathrm{Bt}$ $\mathrm{x}$ Bi y crías Bi para peso al nacer (PN), ganancia diaria promedio predestete (GDP) y PD ajustado a 232 días de edad (Cuadro 2). Las crías nacieron de vacas $\mathrm{C}$ apareadas con toros Ch, Chianina (Ci), Limousin (L), Simmental (Sm), S e I. En general, el PN de las crías F1 Bt $\mathrm{x}$ Bi fue 7\% mayor que el de las crías de sementales Bi. No se detectaron diferencias importantes en los PN de las crías Ci x C, Sm x C, L x C y S x C, pero sí entre los de éstas y los de las Ch x C (Cuadro 2). Los PN de las crías Ch x C, Sm x C, S x C, L x C y Ci x C fueron mayores que los de las crías de toros I en 13, 8, 6, 5 y 4\%, respectivamente. En cuanto al PD ajustado a 232 días de edad, los mayores pesos correspondieron a las crías Sm x C y $\mathrm{Ch} \times \mathrm{C}$, sin que se detectara diferencia importante entre ellas. No se detectaron diferencias significativas entre los PD de crías S x C, L x C, Ci x C e I (Cuadro 2). En PD ajustado a 232 días de edad se encontró una tendencia similar a la observada en PN, las crías Sm x C y Ch x C fueron 13 y $12 \%$ más pesadas al destete que las de padres I, mientras que las crías Sm x $\mathrm{C}$ y $\mathrm{Ch} \times \mathrm{C}$ fueron 8 y $13 \%$ más pesadas al nacer que las de padres $\mathrm{I}^{(12)}$. 
Cuadro 2: Medias de cuadrados mínimos para peso al nacer (PN), ganancia diaria promedio predestete (GDP) y peso al destete ajustado a 205 días (PD205) y 232 días (PD232) de edad de crías Bos indicus y F1 Bos taurus x Bos indicus

\begin{tabular}{|c|c|c|c|c|c|c|}
\hline Grupo genético & $\begin{array}{l}\text { PN } \\
(\mathbf{k g})^{¥}\end{array}$ & $\begin{array}{l}\text { GDP } \\
(\mathbf{k g})^{¥}\end{array}$ & $\begin{array}{l}\text { PD232 } \\
(\mathbf{k g})^{¥}\end{array}$ & $\begin{array}{l}\text { PN } \\
(\mathbf{k g})^{\mathrm{K}}\end{array}$ & $\begin{array}{l}\text { GDP } \\
(\mathbf{k g})^{\mathbf{K}}\end{array}$ & $\begin{array}{l}\text { PD205 } \\
(\mathrm{kg})^{\mathrm{K}}\end{array}$ \\
\hline $\begin{array}{l}\text { Simmental x } \\
\text { Cebú }\end{array}$ & $31.70^{\mathrm{b}}$ & $0.713^{\mathrm{b}}$ & $197.3^{\mathrm{b}}$ & $30.80^{\mathrm{bc}}$ & $0.620^{\mathrm{bc}}$ & $157.80^{c}$ \\
\hline $\begin{array}{l}\text { Charolais x } \\
\text { Cebú }\end{array}$ & $33.08^{c}$ & $0.693^{\mathrm{b}}$ & $193.7^{\mathrm{b}}$ & $31.58^{\mathrm{b}}$ & $0.634^{\mathrm{b}}$ & $159.45^{c}$ \\
\hline $\begin{array}{l}\text { Suizo Pardo x } \\
\text { Cebú }\end{array}$ & $31.24^{\mathrm{b}}$ & $0.644^{\mathrm{a}}$ & $180.5^{\mathrm{a}}$ & $30.24^{\mathrm{c}}$ & $0.602^{b c}$ & $154.44^{\mathrm{bc}}$ \\
\hline $\begin{array}{l}\text { Limousin x } \\
\text { Cebú }\end{array}$ & $30.90^{\mathrm{b}}$ & $0.617^{\mathrm{a}}$ & $174.1^{\mathrm{a}}$ & $30.17^{\mathrm{c}}$ & $0.594^{\mathrm{cd}}$ & $151.15^{\mathrm{bc}}$ \\
\hline Chianina x Cebú & $30.61^{b}$ & $0.605^{\mathrm{a}}$ & $171.0^{\mathrm{a}}$ & $31.17^{\mathrm{b}}$ & $0.602^{b c}$ & $154.56^{\mathrm{bc}}$ \\
\hline Indubrasil & $30.34^{\mathrm{a}}$ & $0.610^{\mathrm{a}}$ & $171.3^{\mathrm{a}}$ & $30.08^{c}$ & $0.564^{\mathrm{d}}$ & $148.91^{\mathrm{b}}$ \\
\hline
\end{tabular}

En el estudio de Martínez ${ }^{(13)}$, con más información que la que se había analizado previamente, se obtuvieron los promedios para PN, GDP y PD ajustado a 205 días que se muestran en el Cuadro 2. Las crías $\mathrm{Ch}$ x $\mathrm{C}$ y $\mathrm{Ci}$ x $\mathrm{C}$ resultaron con $\mathrm{PN}$ mayores que las crías L x C, S x C e I; las crías Sm x C tuvieron PN intermedios. En GDP, las crías Ch x C fueron superiores a las crías L x C e I. No se encontraron diferencias en GDP entre crías Ch x C, Sm x C, Ci x C y S x C, sin embargo, estos grupos genéticos fueron mejores que I. Las crías Ch x C y Sm x C tuvieron mayor PD ajustado a 205 días de edad que las I. No se detectó diferencia significativa entre el PD de las crías I y el de las crías Ci x C, S x C y L x C. Al igual que para $\mathrm{PN}$, las crías $\mathrm{Ch}$ x $\mathrm{C}$ y $\mathrm{Ci}$ x $\mathrm{C}$ mostraron, entre las cruzas $\mathrm{F} 1$, los promedios más alto y más bajo, respectivamente, para PD ajustado a 205 días (Cuadro 2). Con esta información se estimaron los efectos genéticos aditivos individuales para PN, GDP y PD ajustado a 205 días de edad que se muestran en el Cuadro 3. 
Cuadro 3: Efectos genéticos aditivos individuales ${ }^{\mathrm{a}}$ para peso al nacer (PN), ganancia diaria promedio predestete (GDP) y peso al destete ajustado a 205 días de edad (PD205) de cuatro razas Bos taurus y una raza Bos indicus

\begin{tabular}{lccc}
\hline Raza & PN $(\mathbf{k g})$ & GDP $(\mathbf{k g})$ & PD205 $(\mathbf{k g})$ \\
\hline Indubrasil & 1.65 & 51 & 17.9 \\
Charolais & 2.73 & 73 & 15.4 \\
Simmental & 1.22 & 47 & 12.3 \\
Chianina & 1.93 & 15 & 6.3 \\
Suizo Pardo & 0.14 & 15 & 6.1 \\
\hline
\end{tabular}

${ }^{a}$ Expresados como desviación con respecto a la raza Limousin. Martínez ${ }^{(13)}$.

Estos resultados sugieren que el comportamiento de los becerros $\mathrm{C}$ puede ser superado significativamente al utilizar vientres $\mathrm{C}$ en cruzamiento terminal, empleando $\mathrm{Ch}$ o $\mathrm{Sm}$ como raza paterna.

\section{Evaluaciones genéticas de ganado Bos taurus y Bos indicus}

En el Cuadro 4 se presentan los estimadores de heredabilidad y correlaciones genéticas, por raza o población, obtenidos en los estudios realizados por el INIFAP.

Población Simmental-Simbrah. La AMSS fue la primera asociación de criadores de ganado de registro para producción de carne en realizar una evaluación genética nacional (año 2001). Actualmente, con el respaldo del INIFAP, esta asociación publica diferencias esperadas en la progenie para PN, PD, PA, circunferencia escrotal (CE), talla corporal (TC), PP y fertilidad de vaquillas (FV) para Simmental y Simbrah. En las evaluaciones genéticas, así como en los diversos trabajos de investigación que se han realizado con éstas y otras razas (Charolais, Charbray, Brangus Rojo), la FV se define como la probabilidad de que una vaquilla para antes de los 1,281 días (42 meses) de edad; por su parte, la PP se define como la probabilidad de que una vaca tenga una segunda cría o más antes de los seis años de edad, dado que tuvo una cría antes de los tres años de edad. Las diferencias esperadas en la progenie son producto de una evaluación genética multirracial en la que, en general, el modelo animal incluye el grupo contemporáneo (hato-año-estación-sexo de la cría), la edad de la madre al parto (covariable), la proporción de genes Simmental, la heterocigosis y la pérdida por recombinación como efectos fijos. En una evaluación genética multirracial en la que se compararon diferentes variantes del modelo animal se determinó que el modelo más apropiado para la estimación de componentes de varianza de PN, PD y PA fue el que incluyó los efectos genéticos directo y materno y el efecto del ambiente materno permanente, observándose que la exclusión de los efectos maternos (genético y ambiental permanente) o la inclusión de la covarianza entre los efectos genéticos directo y materno causaba 
sobreestimación de la varianza genética aditiva y, en consecuencia, de la heredabilidad; los valores estimados de heredabilidad directa y materna obtenidos con el modelo más apropiado fueron 0.17 y $0.01,0.14$ y 0.02 , y 0.15 y 0.01 para PN, PD y PA, respectivamente (Cuadro $4)^{(14)}$. Adicionalmente, en un análisis multivariado se encontró que las correlaciones genéticas fueron 0.26, 0.26 y 0.62 para PN-PD, PN-PA y PD-PA, respectivamente, lo que sugiere la presencia de efectos pleiotrópicos; sin embargo, dada la magnitud de los valores estimados, se esperaría que la selección para mayor PD resultara en una mayor respuesta correlacionada en PA, comparada con la selección individual para menor PN, lo cual es favorable para los criadores ${ }^{(15)}$.

Cuadro 4: Valores estimados de heredabilidad (en la diagonal dentro de cada población) y correlaciones genéticas

\begin{tabular}{|c|c|c|c|c|c|c|c|c|c|c|c|}
\hline Población & & $\mathbf{P N}_{\mathbf{d}}$ & $\mathbf{P N _ { m }}$ & $\mathbf{P D}_{\mathrm{d}}$ & $\mathbf{P D}_{\mathbf{m}}$ & PAd & $\overline{\mathbf{P A m}}$ & CE & TC & $\mathbf{P P}$ & FV \\
\hline \multirow[t]{10}{*}{ Simmental-Simbrah } & $\mathrm{PN}_{\mathrm{d}}$ & 0.17 & & 0.26 & & 0.26 & & & & & \\
\hline & $\mathrm{PN}_{\mathrm{m}}$ & & 0.01 & & & & & & & & \\
\hline & $\mathrm{PD}_{\mathrm{d}}$ & & & 0.14 & 0 & 0.62 & & & & & \\
\hline & $P D_{m}$ & & & & 0.02 & & & & & & \\
\hline & $\mathrm{PA}_{d}$ & & & & & 0.15 & & 0.36 & 0.47 & & \\
\hline & $\mathrm{PA}_{\mathrm{m}}$ & & & & & & 0.01 & & & & \\
\hline & $\mathrm{CE}$ & & & & & & & 0.35 & 0.59 & -0.24 & -0.65 \\
\hline & TC & & & & & & & & 0.42 & & \\
\hline & PP & & & & & & & & & - & 0.47 \\
\hline & FV & & & & & & & & & & 0.07 \\
\hline \multirow[t]{10}{*}{ Charolais-Charbray } & $\mathrm{PN}_{\mathrm{d}}$ & 0.36 & & & & & & & & & \\
\hline & $\mathrm{PN}_{\mathrm{m}}$ & & 0.13 & & & & & & & & \\
\hline & $\mathrm{PD}_{\mathrm{d}}$ & & & 0.27 & $-0,81$ & & & & & & \\
\hline & $\mathrm{PD}_{\mathrm{m}}$ & & & & 0.15 & & & & & & \\
\hline & $\mathrm{PA}_{d}$ & & & & & 0.30 & & 0.37 & 0.42 & & \\
\hline & $\mathrm{PAm}_{\mathrm{m}}$ & & & & & & 0.12 & & & & \\
\hline & $\mathrm{CE}$ & & & & & & & 0.21 & 0.15 & 0.76 & -0.08 \\
\hline & TC & & & & & & & & 0.25 & & \\
\hline & PP & & & & & & & & & 0.26 & 0.57 \\
\hline & $\mathrm{FV}$ & & & & & & & & & & 0.06 \\
\hline \multirow[t]{10}{*}{ Brangus Rojo } & $\mathrm{PN}_{\mathrm{d}}$ & 0.40 & & 0.41 & & -0.03 & & 0.39 & 0.51 & & \\
\hline & $\mathrm{PN}_{\mathrm{m}}$ & & 0.22 & & & & & & & & \\
\hline & $P D_{d}$ & & & 0.30 & $-0,15$ & 0.68 & & 0.88 & 0.50 & & \\
\hline & $\mathrm{PD}_{\mathrm{m}}$ & & & & 0.06 & & & & & & \\
\hline & $\mathrm{PA}_{d}$ & & & & & 0.30 & & 0.88 & 0.81 & & \\
\hline & $\mathrm{PA}_{\mathrm{m}}$ & & & & & & - & & & & \\
\hline & $\mathrm{CE}$ & & & & & & & 0.18 & 0.97 & & \\
\hline & $\mathrm{TC}$ & & & & & & & & 0.25 & & \\
\hline & PP & & & & & & & & & - & \\
\hline & FV & & & & & & & & & & 0.06 \\
\hline \multirow[t]{5}{*}{ Limousin } & $\mathrm{PN}_{\mathrm{d}}$ & 0.13 & & 0.36 & & 0.58 & & & & & \\
\hline & $\mathrm{PN}_{\mathrm{m}}$ & & 0.15 & & & & & & & & \\
\hline & $P D_{d}$ & & & 0.21 & -0.69 & 0.42 & & & & & \\
\hline & $\mathrm{PD}_{\mathrm{m}}$ & & & & 0.32 & & & & & & \\
\hline & $\mathrm{PA}_{\mathrm{d}}$ & & & & & 0.20 & & & & & \\
\hline \multirow[t]{5}{*}{ Gelbvieh } & $\mathrm{PN}_{\mathrm{d}}$ & 0.30 & & & & & & & & & \\
\hline & $\mathrm{PN}_{\mathrm{m}}$ & & - & & & & & & & & \\
\hline & $P D_{d}$ & & & 0.21 & & & & & & & \\
\hline & $\mathrm{PD}_{\mathrm{m}}$ & & & & - & & & & & & \\
\hline & $\mathrm{PA}_{\mathrm{d}}$ & & & & & 0.45 & & & & & \\
\hline \multirow[t]{2}{*}{ Santa Gertrudis } & $\mathrm{PN}_{\mathrm{d}}$ & 0.06 & & & & & & & & & \\
\hline & $\mathrm{PN}_{\mathrm{m}}$ & & 0.03 & & & & & & & & \\
\hline
\end{tabular}




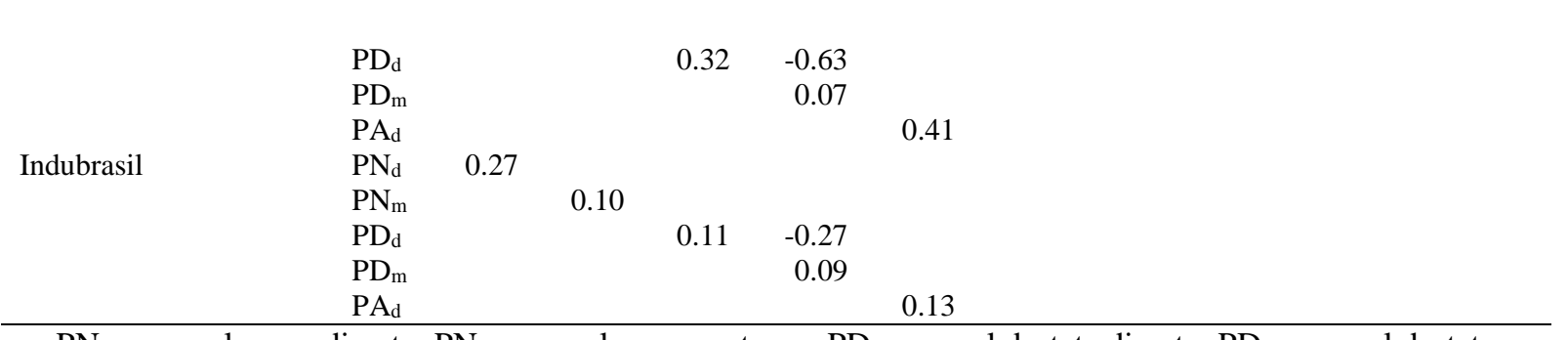

$\mathrm{PN}_{\mathrm{d}}=$ peso al nacer directo, $\mathrm{PN}_{\mathrm{m}}=$ peso al nacer materno, $\mathrm{PD}_{\mathrm{d}}=$ peso al destete directo, $\mathrm{PD}_{\mathrm{m}}=$ peso al destete materno, $\mathrm{PA}_{\mathrm{d}}=$ peso al año directo, $\mathrm{PA}_{\mathrm{m}}=$ peso al año materno, $\mathrm{CE}=$ circunferencia escrotal, $\mathrm{TC}=$ talla corporal, $\mathrm{PP}=$ permanencia productiva, $\mathrm{FV}=$ fertilidad de vaquillas.

Posteriormente, el análisis genético multirracial de toros jóvenes Simmental y Simbrah mostró que los valores estimados de la heredabilidad para PA, CE y TC fueron 0.33, 0.35 y 0.42, mientras que los de las correlaciones genéticas para PA-CE, PA-TC y CE-TC fueron 0.36, 0.47 y 0.59, respectivamente (Cuadro 4), los cuales sugieren la factibilidad de que PA, CE y TC respondan a la selección individual; sin embargo, la selección individual para mejorar una de estas características resultaría en una respuesta correlacionada en las otras $\operatorname{dos}^{(16)}$; por lo tanto, los criadores tendrían que sopesar la conveniencia de producir animales más pesados o con mayor CE al año de edad, ya que la TC de las hembras de reemplazo podría no ser acorde con los recursos disponibles en el sistema de producción.

En lo que concierne a hembras, el análisis genético multirracial de la FV reveló que al comparar la heredabilidad del modelo semental logístico con los modelos lineales, este no condujo a una mejor predicción de los valores genéticos; además, la estimación de los valores genéticos no fue afectada por la distribución de la variable ajustada; sin embargo, la calidad de la información y la conectividad impactaron en la predicción y el ordenamiento de los animales basado en sus valores genéticos ${ }^{(17)}$. Al considerar todos los criterios usados para comparar los modelos, se concluyó que es mejor realizar la evaluación genética de la FV con un modelo animal lineal, dado que la base de datos era lo suficientemente grande $(n=37,390)$, ya que el número de animales con diferencias esperadas en la progenie fue mayor que con los modelos semental, además de que la interpretación de los resultados obtenidos con el modelo semental logístico es más complicada. En dicho estudio se encontró que la heredabilidad de la FV es baja $(0.07)^{(17)}$; sin embargo, los rangos observados de las diferencias esperadas en la progenie, -5.79 a 8.72 para Simmental y -9.56 a 8.84 para Simbrah, indican que puede haber cambio genético en la FV en respuesta a la selección ${ }^{(18)}$.

Adicionalmente, en otros dos estudios se determinó la asociación entre características reproductivas de hembras y de machos, encontrándose en uno de ellos que la correlación genética estimada entre CE y FV (-0.65; Cuadro 4) sugiere que la selección de sementales basada en sus valores genéticos para $\mathrm{CE}$, puede conducir a un mejoramiento de la $\mathrm{FV}$, mientras que la correlación genética detectada entre PP y FV (0.47) sugiere que se puede incrementar la probabilidad de permanencia de las vacas en los hatos cuando éstas son seleccionadas como vaquillas de reemplazo por su valor genético para $\mathrm{FV}^{(19)}$, mientras que 
en el otro estudio se encontró que la edad a primer parto (EPP), el intervalo entre partos (IEP) y el PD acumulado a segundo parto (PDA) están significativamente correlacionados, con valores de 0.42, 0.63 y 0.97 para EPP-IEP, EPP-PDA e IEP-PDA, respectivamente. Adicionalmente, se predijo que la respuesta directa a la selección para IEP y PDA fue mayor que la respuesta correlacionada esperada por la selección para EPP, independiente del número de progenie por semental. La proporción de la superioridad de la selección indirecta a la selección directa fue de 42 y $63 \%$ para EPP-IEP y EPP-PDA, respectivamente ${ }^{(20)}$.

Más recientemente, en un estudio ${ }^{(21)}$ en el que PD se analizó considerándolo como una característica diferente en tres regiones diferentes del país, se encontró que los valores estimados de heredabilidad directa, heredabilidad materna, correlación entre efectos directos y correlación entre efectos maternos fueron diferentes entre regiones, con valores de 0.10 a $0.54,0.44$ a $0.71,0.35$ a 0.69 , y -0.76 a 0.16 , respectivamente, los cuales sugieren la existencia de interacción genotipo-ambiente, ya que se ha considerado que una correlación genética menor que 0.80 indica la existencia de interacción ${ }^{(22)}$, por lo que las evaluaciones genéticas de la raza Simmental deben considerar este factor. Por lo tanto, es importante conocer las condiciones ambientales en donde los toros se seleccionan, dando prioridad a los genotipos superiores en sistemas de producción similares a los del país o la región en donde se criaron.

Por otro lado, en otro estudio se compararon los factores de ajuste aditivos de edad de la madre para PN y PD generados con información de la AMSS con aquellos recomendados por la Federación para el Mejoramiento Genético de los Bovinos de Carne (BIF) de los Estados Unidos de América y los generados por la Asociación Simmental estadounidense (ASA). Para PN no ajustado, la suma de cuadrados (SC) asociada a la edad de la madre fue $8,812 \mathrm{~kg}^{2}$, pero aplicando los factores de corrección de la BIF, la ASA y la AMSS fue 4,595, 1,151 y $184 \mathrm{~kg}^{2}$, respectivamente. Para PD no ajustado, la SC asociada a la edad de la madre fue $110,138 \mathrm{~kg}^{2}$, pero con los factores de corrección de la BIF, la ASA y la AMSS fue $32,0733,241,840$ y $11,245 \mathrm{~kg}^{2}$, respectivamente. Los cuadrados medios del error para PN y PD no ajustados fueron 881 y $11,014 \mathrm{~kg}^{2}$, respectivamente, pero con los factores de corrección estimados fueron 18 y 1,124 $\mathrm{kg}^{2}$. Por lo tanto, se recomienda el uso de los factores de ajuste estimados para ganado Simmental bajo las condiciones ambientales de México, ya que el uso de los factores de ajuste de edad de la madre recomendados por la BIF y la ASA podría sesgar la estimación de los valores genéticos, debido a que las condiciones ambientales de los Estados Unidos de América son diferentes a las de nuestro país. Los factores de ajuste obtenidos para ganado Simmental mexicano son: 1.95, 1.12, 0.41, $0.00 \mathrm{y}$ $0.48 \mathrm{~kg}$, para PN de hembras; y 2.30, 1.21, 0.36, 0.00 y $0.61 \mathrm{~kg}$, para PN de machos hijos de vacas de 2, 3, 4, 5-11 y 12 años de edad; para PD de hembras hijas de vacas de 2, 3, 4, 5-7 y 8-12 años de edad son: 9.30, 5.86, $1.08,0.00$ y $3.04 \mathrm{~kg}$, respectivamente, mientras que para PD de machos hijos de vacas de 2, 3, 4-8 y 9-12 años de edad son: 10.20, 5.55, 0.00 y 3.56 $\mathrm{kg}$, respectivamente ${ }^{(23)}$. 
En una investigación con ganado Simbrah se evalúo la expresión del gen HSP60 en dos momentos, el primero durante la madrugada (AM), cuando la temperatura del ambiente no es elevada y el segundo por la tarde (PM), cuando la temperatura es alta. Los valores $2-\Delta \Delta \mathrm{Ct}$ promedio de las muestras estudiadas en Coahuayana fueron 2.02 en la AM y 1.91 en la PM, en Compostela fueron 3.12 en la AM y 5.16 en la PM, en Tamazula fueron 1.94 en la AM y 2.93 en la PM y en Puerto Vallarta fueron 0.21 en la AM y 0.47 en la PM. De las cuatro localidades de clima tropical estudiadas, en Compostela y Tamazula se observaron diferencias significativas, mientras que en Coahuayana y Puerto Vallarta las diferencias no fueron significativas. Los resultados obtenidos indican que es potencialmente posible identificar animales que podrían incorporarse a programas de mejoramiento genético relacionados con tolerancia al estrés calórico en la raza $\operatorname{Simbrah}^{(24)}$.

Población Charolais-Charbray. La Charolais Charbray Herd Book de México actualmente publica diferencias esperadas en la progenie para las mismas características que evalúa la AMSS, las cuales también son estimadas mediante un modelo animal multirracial. La primera investigación realizada en la población Charolais-Charbray demostró que el modelo animal más apropiado para estimar parámetros genéticos para PN, PD y PA fue el que consistió del efecto genético aditivo directo, el efecto genético aditivo materno, la covarianza entre los efectos genéticos directo y materno y el ambiente materno permanente, ya que este modelo, comparado con otros cinco modelos, redujo sustancialmente -2[logaritmo de la verosimilitud], proporcionando el mejor ajuste para las tres características; los valores estimados de la heredabilidad directa, heredabilidad materna y varianza del ambiente materno permanente como proporción de la varianza fenotípica obtenidos con el modelo más apropiado fueron $0.36,0.13$ y $0.042 ; 0.27,0.15$ y $0.060 ;$ y $0.30,0.12$ y 0.045 para PN, PD y PA, respectivamente (Cuadro 4). En el análisis de cada característica, se observó que el modelo animal más simple, integrado únicamente por el efecto genético aditivo directo, subestimó la varianza genética aditiva directa, pero sobreestimó la varianza residual, en comparación con el modelo más complejo ${ }^{(25)}$.

En un estudio posterior ${ }^{(26)}$ en el que se analizó información de toros jóvenes Charolais y Charbray, CE, TC y PA mostraron ser moderadamente heredables, con valores de 0.21, 0.25 y 0.30 , respectivamente (Cuadro 4). Adicionalmente, las correlaciones genéticas de PA con CE y TC resultaron ser moderadamente fuertes ( 0.37 y 0.42 , respectivamente; Cuadro 4$)$, lo que significa que algunos genes que controlan PA también controlan CE y TC (efecto pleiotrópico), por lo tanto, se espera que toros jóvenes que destacan en PA también destaquen en CE y TC; por el contrario, éstas últimas dos características estuvieron débilmente correlacionadas (0.15; Cuadro 4). Para CE, TC y PA, el estimador de la respuesta directa a la selección basada en cinco descendientes medios hermanos por toro fue $0.38 \mathrm{~cm}, 0.18$ unidades y $8.30 \mathrm{~kg}$, respectivamente. Por otro lado, si se selecciona para mayor PA, se espera que la respuesta correlacionada en la siguiente generación sea $0.16 \mathrm{~cm}$ para CE y 0.08 unidades para TC; por lo tanto, se deduce que la selección indirecta de CE y TC basada en 
PA, no sería tan efectiva como la selección directa para mejorar CE y TC. Finalmente, se observó que las respuestas directa y correlacionada a la selección basadas en 500 descendientes medios hermanos por toro fueron alrededor de dos veces mayores que las basadas en solo cinco descendientes.

Por otro lado, para mejorar genéticamente de la reproducción de las hembras, se desarrolló un prototipo de evaluación genética nacional para la FV Charolais y Charbray, equivalente al desarrollado para la FV Simmental y Simbrah. Con dicho prototipo, el valor estimado de heredabilidad para FV fue 0.06 (Cuadro 4), mientras que los intervalos de las diferencias esperadas en la progenie para Charolais y Charbray fueron -7.94 a 8.22 , y -7.29 a 6.14 , respectivamente, los cuales indican que es factible identificar toros sobresalientes en FV. Adicionalmente, las tendencias genéticas estimadas con este prototipo mostraron un cambio genético favorable en la FV de las dos razas durante el período 2007-2011 ${ }^{(18)}$.

Una de las investigaciones más recientes, en la que se evaluó la asociación entre aspectos reproductivos de machos y hembras, reveló una fuerte correlación genética entre CE y PP (0.76; Cuadro 4), lo que significa que la selección de toros con base en sus diferencias esperadas en la progenie para CE podría inducir un cambio genético favorable en PP; adicionalmente, esta investigación mostró una relación genética importante entre PP y FV (0.57), lo que sugiere la posibilidad de aumentar la probabilidad de que una vaca permanezca en el hato, si a edad temprana se selecciona como vaquilla de reemplazo debido a que cuenta con una alta diferencia esperada en la progenie para FV. En este mismo estudio, los valores estimados de heredabilidad para CE, PP y FV fueron $0.18,0.26$ y 0.11 , mientras que los de la respuesta directa a la selección, asumiendo cinco descendientes medios hermanos por toro, fueron $0.15 \mathrm{~cm}, 0.04$ puntos porcentuales y 0.15 puntos porcentuales, respectivamente; asumiendo este mismo número de descendientes, se espera una respuesta correlacionada a la selección en PP de 0.20 puntos porcentuales en la siguiente generación, si los toros se seleccionan con base en sus diferencias esperadas en la progenie para CE; sin embargo, no se espera respuesta correlacionada en FV; además, para CE, FV y PP, los estimadores de la respuesta directa a la selección basada en 10 descendientes fueron 42.3, 50.0 y $40.0 \%$ mayores, respectivamente, que los estimadores de la respuesta directa a la selección basada en solo 5 descendientes por toro ${ }^{(27)}$.

Población Brangus Rojo. En las evaluaciones genéticas de la raza Brangus Rojo se han incorporado un número importante de características, las mismas que para las razas Simmental, Simbrah, Charolais y Charbray, excepto PP. Un análisis multivariado reciente reveló que el efecto genético aditivo directo de PN, PD, PA y TC es moderadamente heredable $(40,30,30,25 \%$, respectivamente; Cuadro 4), pero el de CE escasamente heredable $(18 \%)$; además, mostró que el efecto genético aditivo materno de PN es moderadamente heredable (22\%), pero el de PD poco heredable (6\%). Concerniente a la relación entre caracteres, dicho análisis permitió deducir que la selección para mayor PD 
puede resultar en una respuesta correlacionada considerable en PN, PA, CE y TC, debido a la magnitud de los estimadores de las correlaciones genéticas de PD con estas cuatro características, la cual fue $0.41,0.68,0.88$ y 0.50 , respectivamente; adicionalmente, permitió determinar que PA y CE, PA y TC, y CE y TC están fuertemente correlacionados, pues proporcionó valores de $0.88,0.81$ y 0.97 , respectivamente ${ }^{(28)}$. Por otro lado, un análisis univariado con un modelo animal lineal mostró que la heredabilidad de FV en Brangus Rojo es baja $(0.06 \text {; Cuadro } 4)^{(29)}$, así como en las poblaciones citadas previamente.

Población Limousin. En las evaluaciones genéticas de la raza Limousin se incluyó PN, PD, PA y CE. En uno de los trabajos de investigación realizados en esta raza, se determinó que el modelo animal más adecuado para PN y PD incluyó los efectos genéticos directo y materno más su covarianza, mientras que para PA solo incluyó el efecto genético directo, observándose que los modelos para PN y PD que no consideraban efectos maternos, sobreestimaban la varianza genética y la heredabilidad. Los efectos genéticos directo y materno de PD resultaron ser más heredables que los de PN ( 0.21 y 0.32 vs 0.13 y 0.15 , respectivamente; Cuadro 4), mientras que los efectos genéticos directos de PD y PA fueron similarmente heredables; el valor estimado de heredabilidad para PA fue 0.20 (Cuadro 4) ${ }^{(30)}$. En otro estudio, en el que se realizó un análisis trivariado, PN, PD y PA resultaron estar moderadamente correlacionados, con valores de 0.36 (PN-PD), 0.58 (PN-PA) y 0.42 (PD$\mathrm{PA})$, por lo que al seleccionar cualquiera de estas características se espera una respuesta correlacionada en las otras dos ${ }^{(31)}$.

Poblaciones Gelbvieh y Santa Gertrudis. En las evaluaciones genéticas de la Asociación Mexicana de Criadores de Ganado Santa Gertrudis y la Asociación de Criadores de Ganado Gelbvieh de la República Mexicana se han calculado diferencias esperadas en la progenie para PN, PD y PA. En una investigación en la que se utilizó información de PN, PD y PA para comparar modelos susceptibles de usarse en la evaluación genética de la raza Gelbvieh, los efectos aleatorios considerados en los modelos fueron: genético directo (D), genético materno (M), la covarianza (C) entre D y M, materno ambiental permanente $(\mathrm{P})$ y residual; del primero al sexto y de acuerdo a los efectos aleatorios incluidos, los modelos se definieron como D, DP, DM, DMP, DMC y DMCP, respectivamente, sin que se detectaran diferencias significativas entre modelos para ninguna de las variables. La inclusión de $\mathrm{C}$ en el modelo subestimó para PN y sobrestimó para PD la heredabilidad directa. Considerando lo anterior y la importancia de los efectos genéticos maternos en ambas características, se sugiere a DMP como el modelo más adecuado para la evaluación genética de PN y PD, pero, para PA, se sugiere a $\mathrm{D}$, considerando la escasa importancia de $\mathrm{M}$ y $\mathrm{P}$ para esta última característica; los valores estimados de heredabilidad directa obtenidos con el modelo más adecuado fueron $0.30,021$ y 0.45 para PN, PD y PA, respectivamente (Cuadro 4) ${ }^{(32)}$. En un estudio posterior, pero en la raza Santa Gertrudis, se observó que los estimadores de la varianza genética aditiva directa, como proporción de la varianza fenotípica, señalan escasa variabilidad genética aditiva directa para PN, en comparación con la variabilidad estimada para PD y PA. Lo 
anterior se refleja en los valores de heredabilidad directa estimados para PN, PD y PA (0.06, 0.32 y 0.41 , respectivamente; Cuadro 4). Por otro lado, los valores estimados de la heredabilidad materna sugieren que muy poca de la variabilidad que existe en PN y PD se debe a efectos genéticos maternos ${ }^{(33)}$.

Población Indubrasil experimental. En un estudio realizado con información de un hato Indubrasil experimental del INIFAP, mantenido en clima tropical húmedo, se observó que para PN y PD el efecto genético directo fue más importante que el efecto genético materno; además, se observó que el efecto materno ambiental permanente no influyó en la expresión de PD. Los valores estimados de heredabilidad directa fueron 0.27, 0.11 y 0.13 para PN, PD y PA, mientras que los de heredabilidad materna fueron 0.10 y 0.09 para PN y PD, respectivamente (Cuadro 4$)^{(34)}$. Por otro lado, en un estudio en el que se analizaron características reproductivas de hembras, IEP, edad a primer servicio y EPP mostraron considerable variación genética y valores de heredabilidad moderados o elevados $(0.13,0.31$ y 0.39 , respectivamente); por el contrario, la duración de la gestación, los días abiertos y el número de servicios por concepción manifestaron poca variación genética, por lo que fueron poco heredables $(0.08,0.03 \text { y } 0.03 \text {, respectivamente })^{(35)}$.

\section{Estimación de la diversidad genética de ganado Bos taurus y Bos taurus x Bos indicus utilizando información genealógica}

La diversidad genética de una población se puede conocer analizando la información contenida en los registros de pedigrí. Esta información genealógica junto con estadísticos basados en las probabilidades de origen de los genes, proveen información valiosa para el estudio de poblaciones que han estado bajo selección por varios años ${ }^{(36)}$. En el análisis de la población mexicana de ganado Simmental de registro se incluyeron animales nacidos entre 1985 y 2014. En 1985 se registraron los primeros animales Simmental nacidos en México. Se encontró que el coeficiente de consanguinidad fue bajo, variando entre $0.68 \%$ para animales nacidos en 2014 y $1.65 \%$ para los nacidos en 1997. Considerando subpoblaciones de cinco años a partir de 1985, el tamaño efectivo de la población aumentó de 134.7 en 19851989 a 186.6 en 2010-2014. El número de fundadores, ancestros y genomas fundadores aumentó de 1985 a 2004, pero disminuyó de 2005 a 2014. La proporción del número efectivo de ancestros en relación con el número efectivo de fundadores y del número efectivo de genomas fundadores en relación con el número efectivo de ancestros para animales nacidos en los períodos 2005-2009 y 2010-2014 sugiere pérdida de diversidad por cuellos de botella y deriva génica en ambos períodos. Por otro lado, un ancestro explicó el $3.4 \%$ de la variabilidad genética total de los animales nacidos en 2010-2014 y 15 ancestros explicaron el $20 \%$ de dicha variabilidad; la contribución genética marginal de cada uno de estos 15 ancestros fue similar $^{(37)}$. 
Para el análisis de la diversidad de la población mexicana de ganado Simbrah de registro se incluyeron animales nacidos entre 1990 y 2014. El coeficiente de consanguinidad fue bajo y con una clara tendencia a disminuir con los años, variando de $0.14 \%$ para animales nacidos en 1990 a $0.03 \%$ para los nacidos en 2014. Considerando subpoblaciones de cinco años a partir de 1990, el tamaño efectivo de la población disminuyó de 79.3 en 1990-1994 a 36.4 en 2010-2014. El número de fundadores, ancestros y genomas fundadores aumentó de 1990 a 2009, pero disminuyó drásticamente en el período de 2005 a 2014. La proporción del número efectivo de ancestros en relación con el número efectivo de fundadores y del número efectivo de genomas fundadores en relación con el número efectivo de ancestros para animales nacidos en el período 2010-2014, sugiere pérdida de diversidad por cuellos de botella y deriva génica. Por otro lado, un ancestro explicó el $0.21 \%$ de la variabilidad genética total de los animales nacidos en 2010-2014 y 10 ancestros explicaron el $1.4 \%$ de dicha variabilidad ${ }^{(38)}$. Para estudiar la evolución de la diversidad genética de la población mexicana de ganado Charolais de registro se utilizó la información de animales nacidos entre 1984 y 2018. El coeficiente de consanguinidad se mantuvo entre 2.1 y $1.3 \%$ durante los 35 años del estudio. El tamaño efectivo de la población aumentó gradualmente de 105.0 en 1984 a 237.1 en 2013, disminuyendo ligeramente a 233.2 en 2018. Se observó un aumento en el número efectivo de ancestros de 1984 a 2008, seguido por una disminución en la última década. El número efectivo de genomas fundadores aumentó de 1984 (130.1) a 2003 (143.7), pero disminuyó en los siguientes 15 años (127.7), resultando en pérdida de alelos de 2004 a 2018. La proporción del número efectivo de ancestros en relación con el número efectivo de fundadores sugiere que la pérdida de diversidad genética en el período de 1999 a 2018 se debió, en parte, a la formación de cuellos de botella en el pedigrí. Además, la proporción del número efectivo de genomas fundadores en relación con el número efectivo de ancestros sugiere pérdida de alelos fundadores por deriva génica ${ }^{(39)}$.

\section{Estudios de asociación de genoma completo para características de crecimiento en ganado Bos taurus}

Se realizaron análisis de asociación con el genoma completo (GC) y por cromosoma (PC) en ganado Simmental ${ }^{(40)}$ y Simbrah $^{(41)}$ de registro, para identificar SNP asociados a características de crecimiento. Se utilizaron genotipos y fenotipos de 967 animales (473 Simmental y 494 Simbrah). Los fenotipos utilizados fueron los valores genéticos deregresados de PN, PD directo (PDD), PD materno (PDM) y PA. Los genotipados se realizaron con paneles de alta densidad. Después del control de calidad, se obtuvieron 105,129 SNP autosómicos. En Simmental, se encontraron 22, 25, 28 y 42 SNP asociados a PN, PDD, PDM y PA, respectivamente. Para PN, se hallaron 18 SNP en el GC y 8 PC; para PDD, 15 SNP tanto en el GC como PC; para PDM, 21 SNP en el GC y 8 PC; para PA, 18 SNP en el GC y 34 PC. Cabe resaltar que 4, 5, 1 y 10 SNP se encontraron tanto en el GC como PC, para PN, PDD, PDM y PA, respectivamente ${ }^{(40)}$. En Simbrah, se encontraron 50, 
29, 18 y 19 SNP asociados a PN, PDD, PDM y PA, respectivamente. Para PN, 24 SNP resultaron significativos en los análisis del GC y 38 SNP en los análisis PC; para PDD, 25 SNP se encontraron en el GC y 9 PC; PDM se asoció a 16 SNP en el GC y 5 PC; para PA, 14 SNP se hallaron en el GC y 12 PC. De los SNP encontrados para PN, PDD, PDM y PA, se hallaron 12, 5, 3 y 7 SNP, respectivamente, tanto en los análisis para el GC como en los análisis $\mathrm{PC}^{(41)}$.

\section{Retos y perspectivas de investigación}

Los objetivos del mejoramiento genético moderno han evolucionado hacia el desarrollo de mejores métodos de evaluación, mediante los cuales se ha podido incrementar la confiabilidad de las estimaciones del valor genético, acortar el intervalo generacional y producir animales cruzados más eficientes ${ }^{(42)}$. A pesar de que desde el 2001 se realizan evaluaciones genéticas en diferentes razas bovinas para producción de carne en México, el mejoramiento genético enfrenta nuevos retos, tanto en su ritmo como en la dirección de sus enfoques. Se ha logrado muy poco o nulo avance en algunas características porque son caras de medir (consumo de alimento residual, producción de metano, tolerancia al estrés calórico), su variación genética es escasa (fertilidad) o la correlación genética es desfavorable entre algunas de estas características ${ }^{(43)}$. Las perspectivas al corto, mediano y largo plazo son las siguientes:

Actualmente, la selección se realiza para un gran número de características para las que se tienen predicciones genéticas y están disponibles a los ganaderos. En el corto plazo es necesario seleccionar animales con base en características de interés a través de índices de selección, como pueden ser los maternos o terminales ${ }^{(44)}$. Se debe incrementar el énfasis en características de la canal; algunas asociaciones de ganado de registro en México han establecido la medición de fenotipos para estas características desde el 2016 y se espera tener predicciones del mérito genético para estas características en corto tiempo. Para las características que se evalúan actualmente se debe contar con estimaciones de las correlaciones genéticas entre ellas y su respuesta correlacionada a la selección. Es necesario mejorar la estructura de la información fenotípica (mayor número de progenie por semental), de tal manera que se establezcan o incrementen las poblaciones de referencia y a mediano plazo se pueda realizar selección genómica ${ }^{(45)}$. En el mediano plazo, es importante considerar nuevos fenotipos (que pueden ser caros y difíciles de medir) que contribuyen a la eficiencia en la producción, dentro de los que se encuentran la producción de metano, tolerancia al estrés calórico, consumo de alimento residual, y características de salud animal que contribuyen a su bienestar, así como la realización de evaluaciones genómicas periódicas para ellos. 
Existe una gran motivación para desarrollar métodos que permitan la comparación entre individuos de diferentes razas, dado las oportunidades comerciales que representa. A mediano y largo plazo, se deberá trabajar en predicción genómica a través de razas, sin embargo, esto será mucho más problemático, ya que diferentes razas pueden exhibir diferentes QTL, puede ocurrir dominancia o epítasis y las frecuencias alélicas pueden variar entre poblaciones ${ }^{(5)}$.

El rol del mejoramiento genético en la producción de carne de bovino es identificar individuos que puedan proveer proteína de alta calidad de forma eficiente, la cual debe de estar balanceada con el ambiente, la emisión de gases de efecto invernadero y la creciente preocupación de los consumidores por el bienestar animal e inocuidad de los alimentos. La producción de carne en México como se desarrolla actualmente no es eficiente, por lo que es necesario que con las herramientas disponibles para el mejoramiento genético se continúe a mediano y largo plazo con la identificación, a través de la selección y cruzamiento, de animales que incrementen la eficiencia productiva y biológica, disminuyendo su huella ambiental, garantizando los estándares más altos de bienestar animal y seguridad alimentaria de la industria.

\section{Conclusiones}

En bovinos $\mathrm{Bt}$, Bi y $\mathrm{Bt} \times \mathrm{Bi}$, en general, se ha logrado: 1) caracterizar productiva (crecimiento, producción de leche, longevidad, productividad total de por vida) y reproductivamente (edad a primer parto, tasas de gestación, parto y destete, kilogramos de becerro destetado por vaca en empadre) animales Bi y Bt x Bi en condiciones tropicales; 2) implementar un modelo de evaluación genética multirracial para las poblaciones SimmentalSimbrah y Charolais-Charbray de registro; 3) determinar la importancia de los efectos maternos (genético y ambiental permanente) en características de crecimiento, lo que permitió la identificación del modelo animal más conveniente para la evaluación genética de PN, PD y PA; 4) estimar la magnitud de la heredabilidad y la correlación genética para características de crecimiento y reproductivas de machos y hembras, incluyendo EPP, IEP y PDA, lo que permite predecir la respuesta directa y correlacionada a la selección basada en diferente número de crías por toro en dichas características; 5) comprobar que la interacción genotipo-ambiente es un factor importante en la expresión de PD en la raza Simmental, por lo que se debe tomar en cuenta en la evaluación genética nacional; 6) desarrollar factores de ajuste de edad de la madre para PN y PD, evitando así el uso de factores de ajuste generados en otros países, lo que podría resultar en estimadores de valores genéticos sesgados; 7) desarrollar un prototipo de evaluación genética nacional para PP y otro para FV, basados en las condiciones ambientales y de manejo de nuestro país; 8) evaluar la expresión del gen HSP60 en bovinos Simbrah expuestos a estrés calórico en el trópico de México; 9) estimar 
la diversidad genética de las poblaciones Simmental, Charolais y Simbrah de registro; y 10) identificar SNP asociados a características de crecimiento en Simmental y Simbrah.

\section{Impactos}

A principios de los ochenta, cuando la mayoría de los ganaderos del trópico mexicano usaban vacas Cebú en la producción de becerros para engorda, se inició el proyecto cuyos resultados se presentan en este documento y mostraron las ventajas de usar vacas cruzadas para producir becerros en los sistemas vaca-cría. En la actualidad, se estima que en más del $50 \%$ de las unidades de producción pecuaria de bovinos para carne del país se usan hembras cruzadas. A raíz de la primera evaluación genética nacional en bovinos productores de carne realizada por investigadores del INIFAP en 2001, con información de la AMSS, las otras asociaciones de criadores de registro comenzaron a realizar sus evaluaciones genéticas nacionales. Actualmente, el INIFAP realiza las evaluaciones genéticas para los criadores de ganado Simmental-Simbrah-Simangus, Charolais-Charbray, Brangus Rojo, Santa Gertrudis, Razas Italianas y Braford, dándoles las herramientas para contribuir al mejoramiento genético del hato nacional, a través de alrededor de 14,000 toros que anualmente venden a productores de registro y comerciales.

\section{Literatura citada:}

1. Cundiff LV, Gregory KE, Schwulst FJ, Koch RM. Effects of heterosis on maternal performance and milk production in Hereford, Angus and Shorthorn cattle. J Anim Sci 1974;38:728-745.

2. Koger M, Peacock FM, Kirk WG, Crockett JR. Heterosis effects on weaning performance of Brahman-Shorthorn calves. J Anim Sci 1975;40:826-822.

3. Gregory KE, Cundiff LV. Crossbreeding in beef cattle: Evaluation of systems. J Anim Sci 1980;51:1224-1242.

4. SAGARPA. Secretaría de Agricultura, Ganadería, Desarrollo Rural, Pesca y Alimentación. Informe sobre la situación de los recursos genéticos pecuarios (RGP). México. 2002.

5. Garrick DJ. The nature, scope and impact of genomic prediction in beef cattle in the United States. Genet Sel Evol 2011;43(17):1-11.

6. Vega MVE, Ríos UA, Montaño BM. Comportamiento productivo hasta el destete de vacas Cebú apareadas con sementales Bos taurus y Bos indicus. Téc Pecu Méx 1996;34:1219. 
7. Ríos UA, Vega MVE, Montaño BM, Lagunes LJ, Rosete FJV. Comportamiento reproductivo de vacas Brahman, Indobrasil y cruzas F1 Angus, Charoláis, Hereford y Suizo Pardo x Cebú y peso al destete de sus crías. Téc Pecu Méx 1996;34:20-28.

8. Ríos UA, Vega MVE, Montaño BM. Causas de desecho y vida productiva de vacas Bos indicus y cruzas Fl Angus, Charolais, Hereford y Suizo Pardo x Cebú. Téc Pecu Méx 1998;36:203-211.

9. Vega-Murillo VE, Ríos-Utrera A, Montaño-Bermúdez M. Herd life and lifetime productivity of Brahman and F1 crossbred Angus, Hereford, Charolais and Brown Swiss x Zebu cows. 7th World Congr Genet Appl Livest Prod 2002; Commun. No. 02-63.

10. Vega-Murillo VE, Ríos-Utrera A, Montaño-Bermúdez M. Stayability of Brahman and F1 crossbred Angus, Hereford, Charolais and Brown Swiss x Zebu cows. 8th World Congr Genet Appl Livest Prod; 2006;8:32-34.

11. Quiroz-Valiente J, Vega-Murillo VE, Ríos-Utrera A, Montaño-Bermúdez M. Milk yield and composition of Brahman and F1 crossbred Angus, Hereford, Charolais and Brown Swiss x Zebu cows. 6th World Congr Genet Appl Livest Prod 1994;17:379-382.

12. Reynoso CO, Villarreal PM, Vazquez PCG. Análisis del crecimiento hasta el destete de animales Bos taurus x Bos indicus criados bajo condiciones tropicales de México. Téc Pecu Méx 1987;25:271-280.

13. Martínez VG. Efectos genéticos aditivos individuales de razas bovinas para características de crecimiento en el trópico [tesis maestría]. Texcoco, Estado de México: Universidad Autónoma Chapingo; 1989.

14. Vega-Murillo VE, Ríos-Utrera A, Montaño-Bermúdez M, Martínez-Velázquez G. Multiple-breed genetic evaluation of growth traits in Simmental and Simbrah cattle. Trop Subtrop Agroecosyst 2012;15(2):403-414.

15. Vega MVE, Torres VA, Manzanilla PC, Baeza RJJ, Borrayo ZA, Martínez VG, Ríos UA, Montaño BM. Correlaciones genéticas entre peso al nacimiento, al destete y al año de edad en ganado Simmental-Simbrah [resumen]. Reunión Nacional de Investigación Pecuaria. Campeche, Camp. 2010:210.

16. Torres-Vázquez JA, Manzanilla-Pech CIV, Borrayo-Zepeda A, Ríos-Utrera A, VegaMurillo VE, Martínez-Velázquez G, Baeza-Rodríguez JJ, Montaño-Bermúdez M. Parámetros genéticos y fenotípicos para peso al año, circunferencia escrotal y talla en ganado Simmental y Simbrah en México. Rev Mex Cienc Pecu 2012;3(3):291-298. 
17. Baeza-Rodríguez JJ, Montaño-Bermúdez M, Vega-Murillo VE, Arechavaleta-Velasco ME. Linear and logistic models for multiple-breed genetic analysis of heifer fertility in Mexican Simmental-Simbrah beef cattle. J Appl Anim Res 2018;46(1):534-540.

18. Baeza-Rodríguez JJ, Vega-Murillo VE, Ríos-Utrera A, Martínez-Velázquez G, Arechavaleta-Velasco ME, Montaño-Bermúdez M. Prototipo de evaluación genética nacional para fertilidad de vaquillas Simmental-Simbrah y Charolais-Charbray. Rev Mex Cienc Pecu 2017;8(3):249-258.

19. Martínez VG, Vega MVE, Román PSI, Ríos UA, Baeza RJJ, Arechavaleta VME, Montaño BM. Correlaciones genéticas entre circunferencia escrotal, fertilidad de vaquillas y permanencia productiva en la población Simmental-Simbrah de México [resumen]. Reunión Nacional de Investigación Pecuaria. Nuevo Vallarta, Nayarit. 2018:268-270.

20. Bejarano-Cabrera DY, Vega-Murillo VE, Montaño-Bermúdez M, Ríos-Utrera Á, Martínez-Velázquez G, Román-Ponce SI, Baeza-Rodríguez JJ, Arechavaleta-Velazco ME. Respuesta directa y correlacionada a la selección para características productivas y reproductivas en ganado Simmental y Simbrah en México [resumen]. Reunión Nacional de Investigación Pecuaria. Nuevo Vallarta, Nay. 2018:283-285.

21. Rosas-García ME, Vega-Murillo VE, Montaño-Bermúdez M, Pescador-Salas N, RománPonce SI, Ríos-Utrera A, Martínez-Velázquez G, Baeza-Rodríguez JJ. Interacción genotipo-ambiente sobre el peso al destete en ganado Simmental [resumen]. Reunión Nacional de Investigación Pecuaria. Tuxtla Gutiérrez, Chiapas. 2019:338-341.

22. Robertson A. The sampling variance of the genetic correlation coefficient. Biometrics 1959;15:469-485.

23. Torres VJA, Montaño BM, Ríos UA, Martínez VG, Vega MVE. Factores de ajuste de edad de la madre para pesos al nacer y al destete en bovinos Simmental [resumen]. Reunión Nacional de Investigación Pecuaria. Querétaro, Qro. 2012:171.

24. Guzmán LF, Martínez-Velázquez G, Villaseñor-González F, Vega-Murillo V, RománPonce S, Montaño-Bermúdez M. Expresión del gen Hsp60 en ganado bovino raza Simbrah expuesto a estrés calórico en el trópico de México [resumen]. XII SIRGEAC Uruguay. Rocha, Uruguay. 2019:149.

25. Ríos-Utrera A, Martínez-Velázquez G, Vega-Murillo VE, Montaño-Bermúdez M. Genetic effects for growth traits of Mexican Charolais and Charbray cattle estimated with alternative models. Rev Mex Cienc Pecu 2012;3(3):275-290. 
26. Ríos Utrera A, Montaño Bermúdez M, Vega Murillo VE, Martínez Velázquez G, Baeza Rodríguez JJ. Genetic parameters of scrotal circumference, frame score and yearling weight of Charolais and Charbray young bulls. Rev Colomb Cienc Pecu 2018;31(3):204-212.

27. Martínez Velázquez G, Ríos Utrera A, Román Ponce SI, Baeza Rodríguez JJ, Arechavaleta Velasco ME, Montaño Bermúdez M, Vega Murillo VE. Genetic correlations between scrotal circumference, heifer fertility and stayability in CharolaisCharbray cattle. Livest Sci 2020;232:103914.

28. Resendiz-Hernández CA, Vega-Murillo VE, Montaño-Bermúdez M, García-Mateos VX Calderón-Chagoya R, Román-Ponce SI, et al. Correlaciones genéticas de características de crecimiento, circunferencia escrotal y talla en bovinos Brangus Rojo en México [resumen]. Reunión Nacional de Investigación Pecuaria. Tuxtla Gutiérrez, Chiapas. 2019:312-315.

29. Baeza RJJ, Vega MVE, Montaño BM, Ríos UA, Román PSI, Martínez VG. Comparación de modelos para el análisis genético de la fertilidad de vaquillas Brangus Rojo [resumen]. Reunión Nacional de Investigación Pecuaria. Tuxtla Gutiérrez, Chiapas. 2019:361-363.

30. Ríos-Utrera A, Vega-Murillo VE, Martínez-Velázquez G, Montaño-Bermúdez M. Comparison of models for the estimation of variance components for growth traits of registered Limousin cattle. Trop Subtrop Agroecosyst 2011;14(2):667-674.

31. Martínez-Velázquez G, Vega-Murillo VE, Ríos-Utrera A, Montaño-Bermúdez M. Parámetros genéticos de análisis univariados y multivariados para peso al nacer, al destete y al año de edad en bovinos Limousin [resumen]. Reunión Nacional de Investigación Pecuaria. Mérida, Yuc. 2008:85.

32. Martínez VG, Vega MVE, Ríos UA, Montaño BM, Borrayo ZA, Baeza RJJ, Manzanilla PC, Torres VJA. Modelos para la evaluación genética de características de crecimiento en bovinos Gelbvieh mexicanos [resumen]. Reunión Nacional de Investigación Pecuaria. León, Gto. 2011:136.

33. Vega MVE, Martínez VG, Montaño BM, Ríos UA. Componentes de varianza y parámetros genéticos para pesos al nacer, al destete y al año de edad de bovinos Santa Gertrudis [resumen]. Congreso de la Asociación de Médicos Veterinarios Zootecnistas Especialistas en Bovinos del Estado de Veracruz. Boca del Río, Ver. 2016:102-104.

34. Ríos-Utrera Á, Hernández-Hernández VD, Villagómez Amezcua-Manjarréz E, ZárateMartínez JP, Villagómez-Cortés A. Direct and maternal genetic effects for growth traits of Indubrazil cattle. Rev Científ FCV-LUZ 2013;23(5):440-447. 
35. Ríos-Utrera Á, Hernández-Hernández VD, Villagómez Amezcua-Manjarréz E, ZárateMartínez JP. Heredabilidad de características reproductivas de vacas Indubrasil. Agron Mesoam 2013;24(2):293-300.

36. Boichard D, Maignel L, Verrier É. The value of using probabilities of gene origin to measure genetic variability in a population. Genet Sel Evol 1997;29:5-23.

37. Ríos-Utrera A, Vega-Murillo VE, Montaño-Bermúdez M, Martínez-Velázquez G, Román-Ponce SI. Genetic diversity assessment of the Mexican Simmental population through pedigree analysis. R Bras Zootec 2018;47:e20160088.

38. Vega MVE, Ríos UA, Montaño BM, Román PSI, Martínez VG. Estudio de la diversidad genética de la población Simbrah mexicana mediante análisis de pedigrí [resumen]. Reunión Nacional de Investigación Pecuaria. Toluca, Edo. de Méx. 2015:210-212.

39. Ríos-Utrera A, Montaño-Bermúdez M, Vega-Murillo VE, Martínez-Velázquez G, BaezaRodríguez JJ, Román-Ponce SI. Genetic diversity evolution in the Mexican Charolais cattle population. Anim Biosci 2021;34(7):1116-1122.

40. Calderón-Chagoya R, Román-Ponce SI, Vega-Murillo VE, García-Ruiz A, MontañoBermúdez M, Ríos-Utrera A, Martínez-Velázquez G. Estudio de asociación de genoma completo para características de crecimiento en ganado Simmental [resumen]. Reunión Nacional de Investigación Pecuaria. Tuxtla Gutiérrez, Chiapas. 2019:342-345.

41. Calderón-Chagoya R, Román-Ponce SI, Vega-Murillo VE, García-Ruiz A, MontañoBermúdez M, Ríos-Utrera A, Martínez-Velázquez G. Estudio de asociación del genoma completo para características de crecimiento en ganado Simbrah [resumen]. Reunión Nacional de Investigación Pecuaria. Tuxtla Gutiérrez, Chiapas. 2019:346-349.

42. Hayes BJ, Lewin HA, Goddard ME. The future of livestock breeding: genomic selection for efficiency, reduced emissions intensity, and adaptation. Trends Genet 2013;29(4):206-214.

43. Ferreira Júnior RJ, Bonilha SFM, Monteiro FM, Cyrillo JNSG, Branco RH, Silva JAIV, Mercadante MEZ. Evidence of negative relationship between female fertility and feed efficiency in Nellore cattle. J Anim Sci 2018;96(10):4035-4044.

44. Golden BL, Garrick DJ, Benyshek LL. Milestone in beef cattle genetic evaluation. J Anim Sci 2009;87(E. Suppl.):E3-E10.

45. Montaldo HH, Casas E, Sterman Ferraz JB, Vega-Murillo VE, Roman-Ponce SI. Opportunities and challenges from the use of genomic selection for beef cattle breeding in Latin America. Anim Front 2012;2(1):23-29. 